\title{
Intelligent Decision Systems Based on Soft Computing Minitrack
}

\author{
Enrique Herrera-Viedma \\ University of Granada \\ viedma@,decsai.ugr.es
}

\author{
Francisco Javier Cabrerizo \\ University of Granada \\ cabrerizo@decsai.ugr.es
}

\author{
Ignacio Javier Pérez \\ University of Cádiz \\ ignaciojavier.perez@uca.es
}

Intelligent decision support systems based on soft computing are of great importance to deal with complex decision environments and involve the design of mathematical models for modeling decision problems, the use of different information technologies (such as the web and social networks), artificial intelligence tools (such as computational intelligence tools), and deep learning methods-based development.

The objective of this minitrack is to attract researchers with an interest in the research area described above. Specifically, we are interested in the contributions towards the development of mathematical models for modeling decision problems, as well as soft computing approaches that are able to deal with complex decision information (such as uncertain and inconsistency information). Topics included in this minitrack are:

- Fuzzy preference modeling in intelligent decision making.

- Intelligent decision-making system applications.

- Consensus in fuzzy multi-agent decision making.

- New models of fuzzy preference modeling.

- Intelligent decision making in complex and dynamic contexts.

- Fuzzy consensus and decision making in web frameworks.

- Consistency issues in preference modeling.

- Intelligent decision-making system for big data.

- Aggregation of preferences in the intelligent decision making.

- Applications of the intelligent decision support systems as supplier selection, ensemble classifiers, portfolio selection, resource allocation, social networks, web.

- Comparison analysis regarding different intelligent decision-making models.

On the one hand, the first paper of this minitrack, "An Adaptive Feedback Mechanism for Consensus Reaching Processes Based on Individuals' Credibility", presents, as a part of a consensus reaching process, a new feedback mechanism based on individuals' credibility. Argumentation is a human feature that allows people to handle discussions by exchanging arguments in a competitive or cooperative fashion to reach a final agreement or just to express an individual position. In such a way, any consensus reaching process should implement some argumentation mechanism in order to imitate the human being way to manage negotiation processes. In addition, the key to believe or refuse arguments given by other people is usually their credibility. To assess the credibility of the individuals could play a crucial role when we carry out a consensus reaching process. Therefore, this paper introduces a segmentation mechanism that divides the individuals into different subsets (very credible, credible and hardly credible). Then, if the individuals of the group do not reach a consensual agreement, this feedback mechanism sends them some recommendations to get their opinions closer. It is based on the assumption that hardly credible individual will need more advice than individuals with high credibility.

On the other hand, the second paper of this minitrack, "A Variance-Based Consensus Degree in Group Decision Making Problems", presents a new consensus measure and perform a comparative study in the context of group decision making problems with fuzzy preference relations. Measures based on statistic variability have been used to measure agreement. Most of them assess disagreement among experts by means of variance as an alternative measure of consensus. In these situations, a high variance is seen as a high disagreement inside the members of the group. In this paper, the standard deviation and the coefficient of variation are used to calculate the consensus levels. As revealed by a subsequent comparative study, the values obtained by this new method are comparable to the values obtained by means of frequently used methods that employ distance functions and aggregation operators, while it turns out to be a simpler application method. Therefore, this index could replace other consensus computations without using distance measures in iterative or non-iterative processes. The implementation of this new index could allow an alternative way to measuring consensus. 